\title{
Perturbations of Weyl-Heisenberg frames
}

\author{
Peter G. Casazza, Ole Christensen and Mark C. Lammers
}

(Received February 19, 2001; Revised August 29, 2001)

\begin{abstract}
We develop a usable perturbation theory for Weyl-Heisenberg frames. In particular, we prove that if $\left(E_{m b} T_{n a} g\right)_{m, n \in \mathbb{Z}}$ is a WH-frame and $h$ is a function which is close to $g$ in the Wiener Amalgam space norm, then also $\left(E_{m b} T_{n a} h\right)_{m, n \in \mathbb{Z}}$ is a WH-frame. We also prove perturbation results for the parameters $a, b$.
\end{abstract}

Key words: Weyl-Heisenberg frame, Gabor frame, perturbation, Riesz basis, frame.

\section{Introduction}

In 1952, Duffin and Schaeffer [10] introduced the notion of a frame for a Hilbert space. A sequence $\left(f_{i}\right)_{i \in I}$ is a frame for a Hilbert space $H$ if there are constants $A, B>0$ satisfying,

$$
A\|f\|^{2} \leq \sum_{i \in I}\left|\left\langle f, f_{i}\right\rangle\right|^{2} \leq B\|f\|^{2},
$$

for all $f \in H$. The constant $A$ (respectively, $B$ ) is a lower (resp. upper) frame bound for the frame. A frame $\left(f_{i}\right)$ can be considered as a "generalized basis": using the fact that the frame operator $S f=\sum\left\langle f, f_{i}\right\rangle f_{i}$ is a bounded invertible operator on $H$, every $f \in H$ can be represented as a convergent series

$$
f=S S^{-1} f=\sum\left\langle f, S^{-1} f_{i}\right\rangle f_{i} .
$$

$\left(f_{n}\right)_{n \in I}$ is a Riesz basic sequence if there exist constants $A, B>0$ such that

$$
A \sum_{i \in I}\left|c_{i}\right|^{2} \leq\left\|\sum_{i \in I} c_{i} f_{i}\right\|^{2} \leq B \sum_{i \in I}\left|c_{i}\right|^{2},
$$

for all finite sequences $\left\{c_{i}\right\}_{i \in I}$. If also $\overline{\operatorname{span}}\left(f_{n}\right)_{n \in I}=H$, then $\left(f_{n}\right)_{n \in I}$ is a Riesz basis. Alternatively, a Riesz basis is a frame which is at the

1991 Mathematics Subject Classification : $42 \mathrm{C} 15$.

The first author was supported by NSF DMS 970618, 0102686 
same time a basis. In particular, all the frame results we prove below have corollaries for Riesz bases.

One of the most important frames for applications, especially signal processing, are the Weyl-Heisenberg frames. Given parameters $a, b \in \mathbb{R}$, we define the translation operator (resp. modulation operator) on $L^{2}(\mathbb{R})$ by

$$
T_{n a} g(t)=g(t-n a), \quad E_{m b} g(t)=e^{2 \pi i m b t} .
$$

For $g \in L^{2}(\mathbb{R})$ and $a, b>0$, we say for short that $(g, a, b)$ is a WeylHeisenberg frame for $L^{2}(\mathbb{R})$ if $\left(E_{m b} T_{n a} g\right)_{m, n \in \mathbb{Z}}$ is a frame for $L^{2}(\mathbb{R})$. Sufficient conditions for $(g, a, b)$ to be a WH-frame can be found in e.g. [2]. For the general theory of Gabor frames we refer the reader to Heil and Walnut [14] or Casazza [1].

WH frames are extremely sensitive to even arbitrarily small changes in the function $g$ and the translation and modulation parameters. For example, $\left(E_{m} T_{n} \chi_{[0,1]}\right)_{m, n \in \mathbb{Z}}$ is a frame for $L^{2}(\mathbb{R})$, but for arbitrary $\epsilon>0$, the functions $\left(E_{m} T_{n} \chi_{[0,1-\epsilon]}\right)_{m, n \in \mathbb{Z}}$ is not. As a result, there are few general theorems on perturbations of $\mathrm{WH}$ frames and those that exist are often very technical in nature (see [11], and the article of Christensen in [13]). In this note we will obtain some very usable perturbation results for WH-frames with only elementary assumptions by using the Wiener Amalgam space norm and by adding continuity assumptions to the function $g$. We will also give examples to show that these results are best possible. Perturbation results not involving the Wiener Amalgam space can be found in the recent paper [16] by Sun and Zhou.

\section{Preliminary Results}

We begin with the perturbation result of Christensen and Heil [7].

Theorem 2.1 If $\left(f_{i}\right)$ is a frame with frame bounds $A, B$ and there exists a constant $R \in[0, A[$ such that for all $f \in H$,

$$
\sum_{i}\left|\left\langle f, f_{i}-g_{i}\right\rangle\right|^{2} \leq R\|f\|^{2}
$$

then $\left(g_{i}\right)$ is a frame with bounds $A\left(1-\sqrt{\frac{R}{A}}\right)^{2}, B\left(1+\sqrt{\frac{R}{B}}\right)^{2}$.

It is known that if $\left(f_{i}\right)$ is a Riesz basis, then the condition in Theorem 2.1 implies that $\left(g_{i}\right)$ is a Riesz basis, cf. [11]. In the rest of this section we 
concentrate on WH-frames. First we recall that the product $a b$ decides which properties a WH-system $(g, a, b)$ can have; for a full discussion we refer to Daubechies' paper [8].

Proposition 2.2 If $(g, a, b)$ is a frame, then

(i) $a b \leq 1$

(ii) $(g, a, b)$ is a Riesz basis if and only if $a b=1$.

Note that the assumption " $(g, a, b)$ a frame" is needed to ensure that $(g, a, b)$ is a Riesz basis for $a b=1$.

To simplify the notation, given a function $g \in L^{2}(\mathbb{R})$ and $a, b \in \mathbb{R}$, we define for $k \in \mathbb{Z}$ the function

$$
G_{k}(t)=\sum_{n \in \mathbb{Z}} g(t-n a) \overline{g(t-n a-k / b)}
$$

It is not difficult to prove that the series defining $G_{k}(t)$ converges absolutely for a.e. $t$. We will need the Weyl-Heisenberg Frame Identity (see [14], Theorem 4.1.5, or [5] for a complete treatment).

Theorem 2.3 (WH-Frame Identity) If $\sum_{n}|g(t-n a)|^{2} \leq B$ a.e. and $f \in$ $L^{2}(\mathbb{R})$ is bounded and compactly supported, then

$$
\begin{aligned}
& \sum_{n, m \in \mathbb{Z}}\left|\left\langle f, E_{m b} T_{n a} g\right\rangle\right|^{2} \\
& =b^{-1} \sum_{k \in \mathbb{Z}} \int_{R} \overline{f(t)} f(t-k / b) \sum_{n} g(t-n a) \overline{g(t-n a-k / b)} d t \\
& =b^{-1} \int_{R}|f(t)|^{2} \sum_{n}|g(t-n a)|^{2} d t \\
& \quad+b^{-1} \sum_{k \neq 0} \int_{R} \overline{f(t)} f(t-k / b) G_{k}(t) d t .
\end{aligned}
$$

We define the Wiener Amalgam space $W\left(L^{\infty}, \ell^{1}\right)$ as the set of functions $g \in L^{2}(\mathbb{R})$ for which for some $a>0$,

$$
\|g\|_{W, a}:=\sum_{n}\left\|g \cdot \chi_{[n a,(n+1) a}\right\|_{\infty}<\infty .
$$

It can be proved that if $\|g\|_{W, a}$ is finite for one value of $a$, it is automatically finite for all $a$. Furthermore, $\|g\|_{W, a}$ defines a norm on $W\left(L^{\infty}, \ell^{1}\right)$. 
We need some elementary facts about the Wiener Amalgam space. These can be found for example in [14], Proposition 4.1.7 and the proof of Theorem 4.1.8. The importance of this space for WH-frames was carefully mapped out by Walnut [17].

Lemma 2.4 Let $g \in W\left(L^{\infty}, \ell^{1}\right)$.

(1) If $0<a \leq b$ then $\|g\|_{W, b} \leq 2\|g\|_{W, a}$.

(2) $\|g\|_{W, a / 2} \leq 2\|g\|_{W, a}$.

(3) Given functions $f, h \in W\left(L^{\infty}, \ell^{1}\right)$,

$$
\sum_{k}\left\|\sum _ { n } \left|T_{n a} f\left\|T_{n a+k / b} h \mid\right\|_{\infty} \leq 4\|f\|_{W, a}\|h\|_{W, a} .\right.\right.
$$

The next result follows from the proof of Theorem 2.3 from [9].

Lemma 2.5 For $g \in L^{2}(\mathbb{R})$ and bounded, compactly supported $f$, we have

$$
\begin{gathered}
\sum_{k \in \mathbb{Z}} \int\left|\overline{f(t)} f(t-k / b) \sum_{n \in Z} g(t-n a) \overline{g(t-n a-k / b)}\right| d t \\
\leq \int|f(t)|^{2} \sum_{k \in \mathbb{Z}}\left|G_{k}(t)\right| d t .
\end{gathered}
$$

\section{Perturbations}

We start with a Proposition which contains the basic tool for our first perturbation result. In light of Theorem 2.1 all we really need to show is that the system $(h-g, a, b)$ has a finite upper frame bound. More specifically:

Proposition 3.1 Suppose $(g, a, b)$ is a WH-frame with frame bounds $A, B$ and let $h \in L^{2}(\mathbb{R})$. If there exists $R<A$ such that

$$
\sum_{k \in \mathbb{Z}}\left|\sum_{n \in \mathbb{Z}}(h-g)(t-n a) \overline{(h-g)(t-n a-k / b)}\right| \leq b R, \text { a.e., }
$$

then $(h, a, b)$ is a Weyl-Heisenberg frame for $H$ with frame bounds $A\left(1-\sqrt{\frac{R}{A}}\right)^{2}, B\left(1+\sqrt{\frac{R}{B}}\right)^{2}$. Moreover, if $(g, a, b)$ is a Riesz basis for $L^{2}(\mathbb{R})$, then $(h, a, b)$ is also a Riesz basis.

Proof. Let $f$ be bounded and compactly supported. By the WH-frame 
Identity and Lemma 2.5 we have:

$$
\begin{aligned}
& \sum_{n, m \in \mathbb{Z}}\left|\left\langle f, E_{m b} T_{n a}(h-g)\right\rangle\right|^{2} \\
& =\frac{1}{b} \sum_{k \in \mathbb{Z}} \int_{\mathbb{R}} \overline{f(t)} f(t-k / b) \sum_{n \in \mathbb{Z}}(h-g)(t-n a) \overline{(h-g)(t-n a-k / b)} d t \\
& \leq \frac{1}{b} \int_{\mathbb{R}}|f(t)|^{2} \sum_{k}\left|\sum_{n \in \mathbb{Z}}(h-g)(t-n a) \overline{(h-g)(t-n a-k / b)}\right| d t \\
& \leq R\|f\|^{2}
\end{aligned}
$$

The set of bounded compactly supported functions is dense in $L^{2}(\mathbb{R})$, so the above estimate actually holds for all functions $f \in L^{2}(\mathbb{R})$. By Theorem 2.1, we have that $(h, a, b)$ is a frame with the given bounds, and $(h, a, b)$ is a Riesz basis if $(g, a, b)$ is a Riesz basis.

In the paper of Jing [15] there is a section concerning perturbations of WH-frames which at first glance appear to be similar to our results. For example, in [15] one of the main perturbation results for WH-frames is that if $(g, a, b)$ is a WH-frame and

$$
\left\|\sum_{k, n \in \mathbb{Z}}|(g-h)(\cdot-n a-k / b)|^{2}\right\|_{\infty}<b A,
$$

then $(h, a, b)$ is also a frame. However, it should be observed that if $a b$ is rational, this condition is only satisfied if $g=h$ a.e., i.e., the result is not useful in that case. Suppose namely that $(g-h)(x) \neq 0$. Since there exist an infinite number of $n, k \in \mathbb{Z}$ such that $n a+\frac{k}{b}=0$, it follows that $\sum_{k, n \in \mathbb{Z}}\left|(g-h)\left(x-n a-\frac{k}{b}\right)\right|^{2}=\infty$. However, Proposition 3.1 above applies for any value of $a b$.

We will now show that our perturbation result works whenever $g, h$ are close in the Wiener Amalgam norm. Note that this result does not require $g$ to be in the Wiener Amalgam space.

Theorem 3.2 Suppose that $(g, a, b)$ is a WH-frame with frame bounds $A, B$. Let $h \in L^{2}(\mathbb{R})$, and assume there exists $R<A$ such that

$$
\|g-h\|_{W, a} \leq \sqrt{\frac{b R}{4}}
$$


Then $(h, a, b)$ is a WH-frame with bounds $A\left(1-\sqrt{\frac{R}{A}}\right)^{2}, B\left(1+\sqrt{\frac{R}{B}}\right)^{2}$. Moreover, if $(g, a, b)$ is a Riesz basis for $L^{2}(\mathbb{R})$, then $(h, a, b)$ is also a Riesz basis.

Proof. Using Lemma 2.4, we have

$$
\begin{aligned}
& \sum_{k \in \mathbb{Z}}\left|\sum_{n \in \mathbb{Z}}(h-g)(t-n a) \overline{(h-g)(t-n a-k / b)}\right| \\
& \quad \leq \sum_{k}\left\|\sum _ { n } \left|T_{n a}(g-h)\left\|T_{n a+k / b}(g-h) \mid\right\|_{\infty}\right.\right. \\
& \quad \leq 4\|g-h\|_{W, a}\|g-h\|_{W, a}=4\|g-h\|_{W, a}^{2} \leq b R .
\end{aligned}
$$

So the result follows from Proposition 3.1.

The condition $R<A$ in Proposition 3.1 can not be relaxed. To see this, fix $\epsilon>0$ and let

$$
g=\chi_{[0,1]}+(1-\epsilon) \chi_{[1,2]}, \quad h=\chi_{[0,2]},
$$

$(g, 1,1)$ is a Riesz basis for $L^{2}(\mathbb{R})$ with lower frame bound $\epsilon^{2}$, since, for any finite sequence of scalars $\left(a_{m n}\right)_{m, n \in \mathbb{Z}}$ we have

$$
\begin{aligned}
& \left\|\sum_{m, n \in \mathbb{Z}} a_{m n} E_{m} T_{n} g\right\| \\
& \geq\left\|\sum_{m, n \in \mathbb{Z}} a_{m n} E_{m} T_{n} \chi_{[0,1]}\right\|-\left\|\sum_{m, n \in \mathbb{Z}} a_{m n} E_{m} T_{n}(1-\epsilon) \chi_{[1,2]}\right\| \\
& =\epsilon\left\|\sum_{m, n \in \mathbb{Z}} a_{m n} E_{m} T_{n} \chi_{[0,1]}\right\|=\epsilon\left(\sum_{m, n \in \mathbb{Z}}\left|a_{m n}\right|^{2}\right)^{1 / 2} .
\end{aligned}
$$

Also,

$$
\sum_{k}\left|\sum_{n \in \mathbb{Z}}(h-g)(x-n) \overline{(h-g)(x-k-n)}\right|=\epsilon^{2}, \quad \text { for a.e. } x .
$$

But $(h, 1,1)$ is not a WH-frame. To see this, observe that

$$
\left\|\sum_{k=0}^{2 n-1}(-1)^{k} T_{k} h\right\|=\left\|\chi_{[0,1]}-\chi_{[2 n-1,2 n]}\right\|=\sqrt{2}=\frac{\sqrt{2}}{2 n} \sum_{k=0}^{2 n-1}\left|(-1)^{k}\right|^{2} .
$$

So $\left(T_{k} h\right)_{k \in \mathbb{Z}}$ is not a Riesz basic sequence in $L^{2}(\mathbb{R})$; thus $(h, 1,1)$ is not a 
Riesz basis, and by Proposition 2.2 this implies that $(h, 1,1)$ is not a frame.

It is an open question which WH-frames are equivalent to compactly supported WH-frames. That is, given a WH-frame $(g, a, b)$, when is it possible to find a frame $(h, c, d)$ with a compactly supported function $h$, such that there exists a bounded invertible operator $T$ for which $T E_{m b} T_{n a} g=$ $E_{m c} T_{n d} h, \forall m, n$ ?

Also, it is another delicate question when we can restrict the function $g$ to a compact subset of $\mathbb{R}$ and still have a WH-frame for $L^{2}(\mathbb{R})$. This question goes directly to the heart of applications where compactly supported WHframes are used. Our next result shows that this is possible whenever $g \in$ $W\left(L^{\infty}, \ell^{1}\right)$.

Corollary 3.3 If $g \in W\left(L^{\infty}, \ell^{1}\right)$ and $(g, a, b)$ is a WH-frame, then there is a natural number $N$ so that $\left(\chi_{[-n a, n a]} g, a, b\right)$ is a WH-frame whenever $n \geq N$.

Proof. We assume that $(g, a, b)$ is a WH-frame with frame bounds $A, B$. Since $g \in W\left(L^{\infty}, \ell^{1}\right)$ we have

$$
\sum_{n \in \mathbb{Z}}\left\|\chi_{[n a,(n+1) a)} g\right\|_{\infty}<\infty .
$$

Since $\left\|\chi_{[-n a, n a]} g-g\right\|_{W, a}=\sum_{|m| \geq n}\left\|\chi_{[m a,(m+1) a)} g\right\|_{\infty}$, we conclude that there is an $N$ so that for all $n \geq N$ we have

$$
\left\|\chi_{[-n a, n a]} g-g\right\|_{W, a}<\sqrt{\frac{b A}{4}} .
$$

The Corollary now follows from Theorem 3.2.

Now we have a considerable strengthening of Proposition 3.1 for the case $a=b=1$.

Theorem 3.4 Let $(g, 1,1)$ be a WH-frame with frame bounds $A, B$. Let $h \in L^{2}(\mathbb{R})$ and $0<\lambda<1$ satisfy

$$
\sum_{n \in \mathbb{Z}}|(g-h)(x+n)| \leq \lambda \sqrt{A} \quad \text { a.e. }
$$

Then $(h, 1,1)$ is a WH-frame for $L^{2}(\mathbb{R})$ with frame bounds

$$
(1-\lambda)^{2} A \quad \text { and } \quad(1+\lambda)^{2} B .
$$


Proof. If $Z$ is the Zak transform, we have

$$
\begin{aligned}
\mid Z(g) & (x, y)-Z(h)(x, y) \mid \\
\quad & \left|\sum_{n \in \mathbb{Z}} g(x+n) e^{2 \pi i n y}-\sum_{n \in \mathbb{Z}} h(x+n) e^{2 \pi i n y}\right| \\
& \leq \sum_{n \in \mathbb{Z}}|(g-h)(x+n)| \leq \lambda \sqrt{A} \leq \lambda|Z g(x, y)| .
\end{aligned}
$$

It follows that,

$$
\begin{aligned}
(1-\lambda) \sqrt{A} & \leq(1-\lambda)|Z(g)(x, y)| \leq|Z(h)(x, y)| \\
& \leq(1+\lambda)|Z(g)(x, y)| \leq(1+\lambda) \sqrt{B} .
\end{aligned}
$$

So $(h, 1,1)$ is a Weyl-Heisenberg frame for $L^{2}(\mathbb{R})$ with the stated frame bounds (see [14], Theorem 4.3.3).

It is easily seen that we can not allow $\lambda=1$ in the inequality in Theorem 3.4. For example, if $g=\chi_{[0,1]}, h=\chi_{[0,2]}$ then $(g, 1,1)$ is an orthonormal basis for $L^{2}(\mathbb{R})$ and as we saw earlier, $(h, 1,1)$ is not a frame. But,

$$
\sum_{n \in \mathbb{Z}}|(g-h)(x+n)|=1 \quad \text { a.e. }
$$

We might hope for an even sharper result with the inequality in Theorem 3.4 changed to

$$
\sum_{n \in \mathbb{Z}}|(g-h)(x+n)|^{2} \leq \lambda A^{\alpha},
$$

for some $0<\alpha \leq 1$. Unfortunately, this fails. For example, let

$$
g=\chi_{[0,1]}
$$

and

$$
h=\frac{1}{2} \chi_{[0,2]} .
$$

Then $(h, 1,1)$ is not a frame (since $\left(T_{n} h\right)_{n \in \mathbb{Z}}$ is not a Riesz basic sequence) while $(g, 1,1)$ is an orthonormal basis for $L^{2}(\mathbb{R})$ (and so $A=B=1$ ). Finally,

$$
\sum_{n \in \mathbb{Z}}|(g-h)(t+n)|^{2}=\left(\frac{1}{2}\right)^{2}+\left(\frac{1}{2}\right)^{2}=\frac{1}{2}=\frac{1}{2} A^{\alpha} .
$$


Now let $(g, a, b)$ be a frame and we will look at perturbations of the modulation and translation parameters to see when we can still be guaranteed to have a WH-frame. The main problem here is that we may not be able to change $a$ or $b$ by any arbitrarily small amount and still get a frame. This follows from a result of Feichtinger and Janssen [12]. They show that there is a function $g \in L^{2}(\mathbb{R})$ so that $(g, a, b)$ has a finite upper frame bound only when $\mathrm{a}$ and $\mathrm{b}$ are rational. Therefore, no matter how close $\left(a^{\prime}, b^{\prime}\right)$ is to $(a, b)$, we still may not have a frame. The next technical difficulty occurs if $a=b=1$. If $(g, 1,1)$ is a WH-frame, then we can never have a general result of the form: $\left|a^{\prime}-a\right|<\epsilon$ implies $\left(g, a^{\prime}, 1\right)$ is a frame since if $a^{\prime}>1$ then $\left(g, a^{\prime}, 1\right)$ is never complete. Despite these strong limitations, we can obtain some satisfactory perturbation results which will guarantee that if the translation parameters are close enough then we will have a frame for all small $b$. In this result, as well as the rest of the results in this section, the price we pay for being able to perturb in one parameter is that the other parameter may change drastically.

Theorem 3.5 Let $g \in W\left(L^{\infty}, \ell^{1}\right)$ with $(g, a, b)$ a WH-frame with frame bounds $A, B$ and let $0<R<b A$. There is an $0<\epsilon \leq \frac{a}{2}$ and $b_{0}=b_{0}(\epsilon)$ so that whenever $\left|a-a^{\prime}\right|<\epsilon$ and

$$
\sum_{n}\left|g(t-n a)-g\left(t-n a^{\prime}\right)\right|^{2} \leq R, \quad \text { a.e. }
$$

then $\left(g, a^{\prime}, b^{\prime}\right)$ is a WH-frame whenever $0<b^{\prime}<b_{0}$.

Proof. If $(g, a, b)$ generates a WH-frame with frame bounds $A, B$ then (see Heil and Walnut [14], the proof of Proposition 4.1.4, page 649)

$$
b A \leq \sum_{n \in \mathbb{Z}}|g(t-n a)|^{2} \leq b B, \text { a.e. }
$$

Using the (reverse) triangle inequality we have

$$
\begin{aligned}
& \sqrt{b A}-\sqrt{R} \\
& \leq\left(\sum_{n}|g(t-n a)|^{2}\right)^{1 / 2}-\left(\sum_{n}\left|g(t-n a)-g\left(t-n a^{\prime}\right)\right|^{2}\right)^{1 / 2} \\
& \leq\left(\sum_{n}\left|g\left(t-n a^{\prime}\right)\right|^{2}\right)^{1 / 2}
\end{aligned}
$$




$$
\begin{aligned}
& \leq\left(\sum_{n}|g(t-n a)|^{2}\right)^{1 / 2}+\left(\sum_{n}\left|g(t-n a)-g\left(t-n a^{\prime}\right)\right|^{2}\right)^{1 / 2} \\
& \leq \sqrt{b A}+\sqrt{R} \text { a.e. }
\end{aligned}
$$

For the rest, we borrow an argument from [14] (the proof of Theorem 4.1.8). Fix $0<\epsilon \leq a / 2$ satisfying

$$
\delta=: 32 \epsilon\|g\|_{W, a}+16 \epsilon^{2}<[\sqrt{b A}-\sqrt{R}]^{2} .
$$

Now let $N$ be so large that

$$
\sum_{|n| \geq N}\left\|g \cdot \chi_{[a n, a(n+1))}\right\|_{\infty}<\epsilon
$$

Let $g_{0}=g \cdot \chi_{[-a N, a N]}$ and $g_{1}=g-g_{0}$, so that $\left\|g_{1}\right\|_{W, a}<\epsilon$. Now if

$$
b^{\prime} \leq \frac{1}{4 a N}=b_{0}
$$

then $\left(\right.$ with $\left.G_{k}^{\prime}(t):=\sum_{n} T_{n a^{\prime}} g(x) \cdot T_{n a^{\prime}+k / b^{\prime}} \overline{g(x)}\right)$

$$
\begin{aligned}
& \sum_{k \neq 0}\left\|G_{k}^{\prime}(t)\right\|_{\infty}=\sum_{k \neq 0}\left\|\sum_{n} T_{n a^{\prime}} g \cdot T_{n a^{\prime}+k / b^{\prime}} \bar{g}\right\|_{\infty} \\
& \leq \sum_{k \neq 0}\left\|\sum _ { n } \left|T_{n a^{\prime}} g\left\|T_{n a^{\prime}+k / b^{\prime}} g \mid\right\|_{\infty}\right.\right. \\
& =\sum_{k \neq 0}\left\|\sum _ { n } \left|T_{n a^{\prime}} g_{0}+T_{n a^{\prime}} g_{1}\left\|T_{n a^{\prime}+k / b^{\prime}} g_{0}+T_{n a^{\prime}+k / b^{\prime}} g_{1} \mid\right\|_{\infty}\right.\right. \\
& \leq \sum_{k \neq 0}\left\|\sum_{n}\left|T_{n a^{\prime}} g_{0}\left\|T_{n a^{\prime}+k / b^{\prime}} g_{0}\left|\left\|_{\infty}+\sum_{k \neq 0}\right\| \sum_{n}\right| T_{n a^{\prime}} g_{0}\right\| T_{n a^{\prime}+k / b^{\prime}} g_{1}\right|\right\|_{\infty} \\
& \quad+\sum_{k \neq 0}\left\|\sum_{n}\left|T_{n a^{\prime}} g_{1}\left\|T_{n a^{\prime}+k / b^{\prime}} g_{0}\left|\left\|_{\infty}+\sum_{k \neq 0}\right\| \sum_{n}\right| T_{n a^{\prime}} g_{1}\right\| T_{n a^{\prime}+k / b^{\prime}} g_{1}\right|\right\|_{\infty} \\
& \leq 0+8\left\|g_{0}\right\|_{W, a^{\prime}}\left\|g_{1}\right\|_{W, a^{\prime}}+4\left\|g_{1}\right\|_{W, a^{\prime}}^{2} .
\end{aligned}
$$

Now, since $\frac{a}{2} \leq a^{\prime} \leq 2 a$, we can continue our inequality using Lemma 2.4,(1) and (2) to get:

$$
\sum_{k \neq 0}\left\|G_{k}^{\prime}(t)\right\|_{\infty}=\sum_{k \neq 0}\left\|\sum_{n} T_{n a^{\prime}} g \cdot T_{n a^{\prime}+k / b^{\prime}} \bar{g}\right\|_{\infty}
$$




$$
\begin{aligned}
& \leq 8\left\|g_{0}\right\|_{W, a^{\prime}}\left\|g_{1}\right\|_{W, a^{\prime}}+4\left\|g_{1}\right\|_{W, a^{\prime}}^{2} \\
& \leq 32\left\|g_{0}\right\|_{W, a}\left\|g_{1}\right\|_{W, a}+16\left\|g_{1}\right\|_{W, a}^{2} \\
& \leq 32 \epsilon\|g\|_{W, a}+16 \epsilon^{2}=\delta .
\end{aligned}
$$

It follows by Lemma 2.5 that if $\left|a-a^{\prime}\right|<\epsilon$ and $0<b^{\prime} \leq b_{0}$ then for all bounded, compactly supported functions $f \in L^{2}(\mathbb{R})$ we have

$$
\begin{aligned}
& \frac{1}{b^{\prime}}\left|\sum_{k \neq 0} \int_{\mathbb{R}} \overline{f(t)} f\left(t-k / b^{\prime}\right) G_{k}^{\prime}(t) d t\right| \\
& \quad \leq \frac{1}{b^{\prime}} \sum_{k \neq 0}\left\|G_{k}^{\prime}(t)\right\|_{\infty} \int_{\mathbb{R}}|f(t)|^{2} d t \leq \frac{1}{b^{\prime}} \delta\|f\|^{2} .
\end{aligned}
$$

Also, from the first part of the proof, for all $f$ as above we have,

$$
\begin{aligned}
& \frac{1}{b^{\prime}}(\sqrt{b A}-\sqrt{R})^{2}\|f\|^{2} \\
& \quad \leq \frac{1}{b^{\prime}} \int_{\mathbb{R}}|f(t)|^{2} \sum_{n \in \mathbb{Z}}\left|g\left(t-n a^{\prime}\right)\right|^{2} \leq \frac{1}{b^{\prime}}(\sqrt{b A}+\sqrt{R})^{2}\|f\|^{2} .
\end{aligned}
$$

Finally, the WH-Frame Identity yields,

$$
\begin{aligned}
\sum_{m, n \in \mathbb{Z}}\left|\left\langle f, E_{m b^{\prime}} T_{n a^{\prime}} g\right\rangle\right|^{2}= & \frac{1}{b^{\prime}} \int_{\mathbb{R}}|f(t)|^{2} \sum_{n \in \mathbb{Z}}\left|g\left(t-n a^{\prime}\right)\right|^{2} d t \\
& +\frac{1}{b^{\prime}} \sum_{k \neq 0} \int_{\mathbb{R}} \overline{f(t)} f\left(t-k / b^{\prime}\right) G_{n}^{\prime}(t) d t .
\end{aligned}
$$

Putting this altogether we have that

$$
\begin{aligned}
& \frac{1}{b^{\prime}}\left[(\sqrt{b A}-\sqrt{R})^{2}-\delta\right]\|f\|^{2} \\
& \quad \leq \sum_{m, n \in \mathbb{Z}}\left|\left\langle f, E_{m b^{\prime}} T_{n a^{\prime}} g\right\rangle\right|^{2} \leq \frac{1}{b^{\prime}}\left[(\sqrt{b A}+\sqrt{R})^{2}+\delta\right]\|f\|^{2} .
\end{aligned}
$$

Since this inequality holds for all bounded compactly supported functions $f \in L^{2}(\mathbb{R})$, it holds for all $f \in L^{2}(\mathbb{R})$, which completes the proof.

A general setting where the conditions of Theorem 3.5 will hold is when $g$ is continuous. This is just enough to offset the Feichtinger-Janssen example [12]. 
Corollary 3.6 If $g \in W\left(L^{\infty}, \ell^{1}\right)$ is continuous and $(g, a, b)$ is a frame, then there is $a \delta>0$ and $a b_{0}>0$ so that $\left(g, a^{\prime}, b^{\prime}\right)$ is a WH-frame whenever

$$
\left|a-a^{\prime}\right|<\delta
$$

and $0<b^{\prime}<b_{0}$.

Proof. We just need to verify that the conditions of Theorem 3.5 hold. Fix $R<b A$. Since $g \in W\left(L^{\infty}, \ell^{1}\right)$, we can choose a natural number $n_{0}$ so that

$$
\left\|\left(1-\chi_{\left[a\left(-n_{0}+1\right), a n_{0}\right]}\right) g\right\|_{W, a}<\frac{R}{3} .
$$

Since $g$ is continuous on the compact set $\left[-a n_{0}, a\left(n_{0}+1\right)\right]$, it is uniformly continuous there. In particular, there is a $\delta>0$ so that if $x, y \in$ $\left[-a n_{0}, a\left(n_{0}+1\right)\right]$ then

$$
|x-y| \leq \delta, \Rightarrow|g(x)-g(y)|^{2}<\frac{R}{3\left(2 n_{0}+2\right)} .
$$

Let $\epsilon=\frac{\delta}{n_{0}}$. Now, if $\left|a-a^{\prime}\right|<\epsilon$ and then for all $-n_{0} \leq n \leq n_{0}-1$ we have

$$
\left|(t-n a)-\left(t-n a^{\prime}\right)\right|=|n|\left|a-a^{\prime}\right|<|n| \epsilon=\frac{|n|}{n_{0}} \delta \leq \delta .
$$

Hence, for $t \in[0, a]$,

$$
\left|g(t-n a)-g\left(t-n a^{\prime}\right)\right|^{2}<\frac{R}{3\left(2 n_{0}+2\right)},
$$

It follows that

$$
\begin{aligned}
& \sum_{n}\left|g(t-n a)-g\left(t-n a^{\prime}\right)\right|^{2} \\
& =\sum_{n=-n_{0}}^{n_{0}+1}\left|g(t-n a)-g\left(t-n a^{\prime}\right)\right|^{2}+\sum_{\substack{n<-n_{0} \\
n>n_{0}+1}}\left|g(t-n a)-g\left(t-n a^{\prime}\right)\right|^{2} \\
& \leq\left(2 n_{0}+2\right) \frac{R}{3\left(2 n_{0}+2\right)}+2\left\|\left(1-\chi_{\left[a\left(-n_{0}+1\right), a n_{0}\right]}\right) g\right\|_{W, a} \\
& <\frac{R}{3}+\frac{2 R}{3}=R .
\end{aligned}
$$

The Corollary now follows by Theorem 3.5. 
We now have immediately the corresponding result for compactly supported functions.

Corollary 3.7 If $(g, a, b)$ is a WH-frame where $g$ is compactly supported and continuous, then there is $a \delta>0$ and $a b_{0}>0$ so that $\left(g, a^{\prime}, b^{\prime}\right)$ is a WH-frame whenever

$$
\left|a-a^{\prime}\right|<\delta
$$

and $0<b^{\prime}<b_{0}$.

Continuity is necessary in the preceding results. A trivial example occurs if we consider $\left(\chi_{[0,1]}, 1,1\right)$ since no matter how close we have $a^{\prime}$ to $a$, if $a<a^{\prime}$, we cannot have a frame for any $b$ since a necessary condition for $(g, a, b)$ to form a WH-frame with frame bounds $A, B$ is that $b A \leq$ $\sum_{n \in \mathbb{Z}}|g(t-n a)|^{2} \leq b B$, a.e. In light of this, it is more natural to ask for $\left(g, a^{\prime}, b^{\prime}\right)$ to form a frame for $0<a-a^{\prime}<\epsilon$, and all small $b^{\prime}$. But again, the above results will fail without the assumption of continuity. For example, we can let

$$
\begin{aligned}
& E_{1}=\left[0,1-\frac{1}{16}\right), \\
& E_{2}=\bigcup_{n=2}^{\infty}\left[1-\frac{1}{2^{2 n}}, 1-\frac{1}{2^{2 n+1}}\right),
\end{aligned}
$$

and

$$
E_{3}=\bigcup_{n=2}^{\infty}\left[2-\frac{1}{2^{2 n+1}}, 2-\frac{1}{2^{2(n+1)}}\right) .
$$

Let $F=E_{1} \cup E_{2} \cup E_{3}$ and $g=\chi_{F}$. Then it is immediate that $(g, 1,1)$ is an orthonormal basis for $L^{2}(\mathbb{R})$. Now, if

$$
1-\frac{1}{2^{2 n+1}}<a^{\prime}<1-\frac{1}{2^{2(n+1)}}
$$

then for all

$$
1-\frac{1}{2^{2 n+1}}<t \leq a^{\prime}
$$

we have that $g(t)=0$, and for $n \geq 1, t-n a^{\prime}<0$ so $g\left(t-n a^{\prime}\right)=0$. Also, for $n \geq 2$ we have that $2<t+n a^{\prime}$ and so $g\left(t+n a^{\prime}\right)=0$. Finally, for $n=1$ 
we have that

$$
\begin{aligned}
2-\frac{1}{2^{2 n}} & =1-\frac{1}{2^{2 n+1}}+1-\frac{1}{2^{2 n+1}} \\
& \leq t+a^{\prime}<1-\frac{1}{2^{2(n+1)}}+1-\frac{1}{2^{2(n+1)}}=2-\frac{1}{2^{2 n+1}} .
\end{aligned}
$$

Hence, $g\left(t+a^{\prime}\right)=0$. It follows that

$$
\sum_{n \in \mathbb{Z}}\left|g\left(t-n a^{\prime}\right)\right|^{2}=0, \quad \text { for all } 1-\frac{1}{2^{2 n+1}}<t \leq a^{\prime} .
$$

In particular, $\left(g, a^{\prime}, b\right)$ is not a frame for all $0<b$. It follows that, given any $\epsilon>0$, there is an interval of points $a^{\prime}$ with $0<a-a^{\prime}<\epsilon$ so that $\left(g, a^{\prime}, b\right)$ is not a frame for all $0<b$.

Acknowledgment The authors would like to thank the referees for their good comments and for providing extra references.

\section{References}

[1] Casazza P.G., Modern Tools for Weyl-Heisenberg (Gabor) Frame Theory. Advances in Imaging and Electron Physics 115 (2000), 1-127.

[2] Casazza P.G. and Christensen O., Weyl-Heisenberg frames for subspaces of $L^{2}(\mathbf{R})$. Proc. Amer. Math. Soc. 129 (2001), 145-154.

[3] Casazza P.G. and Christensen O., Perturbation of operators and applications to frame theory. J. Fourier Anal. Appl. 3 (1997), 543-557.

[4] Casazza P.G., Christensen O. and Janssen A.J.E.M., Weyl-Heisenberg frames, translation invariant systems and the Walnut Representation. J. Funct. Anal. 180, No.1 (2001), 85-147.

[5] Casazza P.G. and Lammers M.C., Analyzing the Weyl-Heisenberg frame identity. Appl. Comp. Harm. Anal. 12, No.2 (2002), 171-178.

[6] Christensen O., Frames, bases, and discrete Gabor/wavelet expansions. Bull. Amer. Math. Soc. 38, No.3 (2001), 273-291.

[7] Christensen O. and Heil C., Perturbation of Banach frames and atomic decomposition. Math. Nach. 185 (1997), 33-47.

[8] Daubechies I., The wavelet transformation, time-frequency localization and signal analysis. IEEE Trans. Inform. Theory 36 (1990), 961-1005.

[9] Deng B., Schempp W., Xiao C. and Wu Z., On the existence of Weyl-Heisenberg and affine frames. Preprint, 1997.

[10] Duffin R.J. and Schaeffer A.C., A class of non-harmonic Fourier series. Trans. Amer. Math. Soc. 72 (1952), 341-366.

[11] Favier S.J. and Zalik R.A., On the stability of frames and Riesz bases. Applied and Computational Harmonic Analysis 2 (1995), 160-173. 
[12] Feichtinger H.G. and Janssen A.J.E.M., Validity of WH-frame bound conditions depends on lattice parameters. Appl. Comp. Harm. Anal. 8, No.1 (2000), 104-112.

[13] Feichtinger H. and Strohmer T. (eds.), Gabor Analysis and Algorithms: Theory and Applications. Birkhauser, Boston (1998).

[14] Heil C. and Walnut D., Continuous and discrete wavelet transforms. SIAM Review 31 (1989), 628-666.

[15] Jing Z., On the stability of wavelet and Gabor frames (Riesz bases). J. Fourier Anal. Appl. 5, No.1 (1999), 105-125.

[16] Sun W. and Zhou X.X., On the stability of Gabor frames. Adv. in Appl. Math. 26 (2001), 181-191.

[17] Walnut D., Weyl-Heisenberg wavelet expansions: existence and stability in weighted spaces. Ph.D. thesis, University of Maryland, College Park, MD, 1989.

[18] Walnut D., Gabor-type expansions in weighted spaces on regular and irregular lattices. Preprint.

Peter G. Casazza

Department of Mathematics

University of Missouri-Columbia

Columbia, MO 65211, U.S.A.

E-mail: pete@math.missouri.edu

Ole Christensen

Department of Mathematics

Technical University of Denmark

2800 Lyngby, Denmark

E-mail: Ole.Christensen@mat.dtu.dk

Mark C. Lammers

Department of Mathematics

University of South Carolina

Columbia, SC 29208, U.S.A.

E-mail: lammers @math.sc.edu 